\title{
Kinetics of self-organization of polyampholyte nanoparticles in solutions
}

\author{
H B BOHIDAR \\ School of Physical Sciences, Jawaharlal Nehru University, New Delhi 110 067, India
}

\begin{abstract}
An appropriate composition of a binary solvent, water-ethanol, provides the necessary thermodynamic environment for the polyampholyte (gelatin) molecules to form self-assembled nano-clusters having fractal dimension, $\approx 2 \cdot 6$, in the bulk (3-D). The aggregation in the bulk of the solution appears to be an anomalous process and could be explained through Smoluchowski aggregation model. It gives a diffusion limited aggregation (DLA) type fractal dimension to the cluster in bulk, but shows extremely low polydispersity, which in fact is a signature of a slowly growing reaction limited cluster aggregation (RLA) process. Experimental results obtained from light scattering and electrophoresis experiments enable us to probe the kinetics of such growth processes.
\end{abstract}

Keywords. Gelatin aggregation; self-organization; fractal dimension; light scattering; DLA.

\section{Introduction}

The phenomenon of self-organization and aggregation are of significant interest to biology, immunology, polymer science, colloidal chemistry, metallurgy and nucleation processes close to phase transitions. Such aggregations in polyelectrolytic solutions produce polymer-rich solvated condensates, called coacervates that have rich biological implications (Bungenberg de Jong 1949; Oparin 1957). Self-organization and aggregation processes have been described through diffusion limited cluster aggregation (DLA) (Witten and Sander 1981, 1983), reaction limited cluster aggregation (RLA) (Feder et al 1984, 1985), random sequential adsorption (RSA) (Evans 1993) etc. The quantification of these aggregates is done through Hausdorff mass fractal dimension, $d_{\mathrm{f}}$, which is much smaller than the corresponding Eucleadian dimension $(D)$. The stochastic self-similarity and scaling of the random fractals is ascertained from the fractal dimension, $d_{\mathrm{f}}$, which is defined as (Birdi 1991)

$$
d_{\mathrm{f}}(s)=\lim _{\varepsilon \rightarrow 0} \frac{\ln M(\varepsilon)}{\ln (1 / \varepsilon)},
$$

where $s$ is a subset of $N$-dimensional space occupied by the fractal object and $M(\varepsilon)$ the number of $N$-dimensional cubes (of side $\varepsilon$ ) required to cover the subset, $s$. In this picture, the density-density correlation function of an assembly is given by

$$
g(r)=\langle\rho(r) \rho(0)\rangle \sim r^{-\mathrm{A}},
$$

where $A=D-d_{\mathrm{f}}$. Diffusion driven processes rapidly coagulate to yield DLA clusters of fractal dimension, $d_{\mathrm{f}} \cong 1.7$ for

(bohi0700@mail.jnu.ac.in)
$D=2$ and 2.6 for $D=3$ which is strongly supported by simulations and experimental results. On the other hand, RLA clusters grow rather slowly because of reduced collision probability between particles and the growth is through inter-particle reactivity involving equilibrium constant. The corresponding fractal dimensions are much higher than that of DLA clusters. Though the crossover from DLA to RSA has been observed, the intermediate region remains relatively unexplored (Di Biasio et al 1994).

Adhesion of macromolecules onto surfaces, polymer chain reactions, association of non-crystalline granular materials and arrangement of nano-structures, quantum memory devices, biosensors on substrates, car parking etc fall into the category of random sequential adsorption process (RSA). These processes are generated with sequential addition of particles at a constant rate to a substrate where overlapping and leaving the cluster once a part is forbidden. Brilliantov et al (1996) have shown that geometric and kinetic features of RSA clusters for a system showing polydispersity in size are governed by the smallest size particles but prior to this an intermediate asymptotic regime prevails. Brilliantov et al (1996) assumed the particle size distribution to be continuous and represented it as a power-law distribution function, $P(R)$, given by

$$
P(R)=\left\{\begin{array}{cl}
\alpha R^{\alpha-1} ; & R \leq R_{\max } \\
0 ; & R>R_{\max }
\end{array}\right.
$$

The system is assumed to have a very large polydispersity in size with the maximum size given by $R_{\max }$. The geometrical structure of the resultant RSA cluster evolving from a system with characteristics defined by particle 
size distribution as in (1) is fully governed by the spatial correlations inside the system, which is accounted for by the parameter, $\alpha(\alpha>0)$. As $\alpha$ increases from 0 to $\infty, d_{\mathrm{f}}$ reduces from 2 to $1 \cdot 305$. For smaller $\alpha$ fractal clusters are formed while the Apollonian packing limit is reached as $\alpha \rightarrow \infty$.

The theoretical concepts recapitulated above necessitate three requirements: (i) system has a wide size particle distribution, (ii) the fractal state is an intermediate phase and (iii) the final state is a state where the smallest particle size wins giving rise to a regular cluster. Gelatin, which is a low charge density polypeptide, dissolves in water above circa. $50^{\circ} \mathrm{C}$ and ethanol is a non-solvent. Thus, addition of ethanol to an aqueous solution of gelatin creates a hostile thermodynamic environment (marginal solvent) for this polyelectrolyte compelling the gelatin molecules to aggregate, and to undergo several conformational transitions (Mohanty and Bohidar 2005). At a threshold ethanol to water composition ratio the bulk of the matter is expelled out of the marginal solvent along with its hydration water into a separate equilibrium phase called coacervates (Mohanty and Bohidar 2003). The polyions do not precipitate out of the solvent because of entropy gain achieved by random mixing of polyions in the coacervate phase, which is the final equilibrium state. Though multivalent salt induced complexation of gelatin has been reported in the past (Bungenberg de Jong 1949), the universality of growth dynamics of this biopolymer in the bulk driven by desolvation of the gelatin molecules was never established conclusively which is the main objective of this paper.

\section{Materials and methods}

An aqueous solution of gelatin $(1 \% \mathrm{w} / \mathrm{v}$, less than the gelation concentration (Bohidar and Jena 1993), $\approx 2 \%$ w/v) obtained from Merck, Germany (Type-B, bovine skin extract devoid of $E$ coli and liquefier presence, bloom strength $=75, \quad \mathrm{pI}=4.9$, nominal molecular weight $=$ $100,000 \mathrm{Da}$, estimated by SDS/PAGE), was prepared by dissolving the gelatin powder in deionized water at $50^{\circ} \mathrm{C}$ with ionic strength set to $0 \cdot 1 \mathrm{M} \mathrm{NaCl}$. The solution $\mathrm{pH}$ was adjusted to $\mathrm{pH}=5$. When cooled to room temperature, it produced a clear transparent solution. This stock solution was titrated with ethanol until turbidity was noticed. The particle sizing measurements were done by dynamic laser light scattering (DLS) technique, using a 9000-AT digital correlator bought from Brookhaven Instruments Corp. (BIC), USA and a homemade goniometer. The excitation source was a He-Ne laser emitting at a wavelength of $632.8 \mathrm{~nm}$ in linearly polarized single frequency mode, which was focused on the sample cell and scattered light was detected by a photo-multiplier tube (Hamamatsu) and the signal was converted into intensity auto-correlation function by the correlator that was operated in multi-tau mode. During the course of size measurements the intensity of scattered light was monitored continuously as a function of time over a period of $8 \mathrm{~h}$. The scattering angle was fixed at $90^{\circ}$ and the data analysis was done using CONTIN software provided by Brookhaven Instruments. This technique was used to get the size of the particles. The DLS experiment measures the time correlation function, $g_{2}(t)$, of the scattered intensity, $I(t)$, at a given $q$, scattering vector, is defined by (Bohidar 2002)

$$
g_{2}(t)=\frac{\left\langle I\left(t^{\prime}\right) I\left(t^{\prime}+t\right)\right\rangle}{\left\langle I\left(t^{\prime}\right)\right\rangle^{2}},
$$

where $q=(4 \pi n / \lambda) \sin (\theta / 2), n$ is the refractive index of the solution, $\theta$ the scattering angle, and $\lambda$ the wavelength of light source in medium. The intensity correlation function, $g_{2}(t)$, is related to the scattered field auto-correlation function, $g_{1}(t)$, through the Siegert relation (Bohidar 2002)

$$
g_{2}(t)=A+B\left|g_{1}(t)\right|^{2},
$$

where $A$ defines the baseline of the correlation function as

$$
\left|g_{2}(t)\right|_{t \rightarrow \infty}=A,
$$

and $B$ is the spatial coherence factor. The ratio $(B / A)$ is the signal modulation and better data quality demands $(B / A) \geq$ $50 \%$. For solutions containing particles undergoing Brownian motion (i.e. polymer or colloidal solutions), the field autocorrelation function, $g_{1}(t)$, is given as

$$
g_{1}(t)=\sum_{i} A_{i} \exp \left(-\Gamma_{i} t\right)
$$

where $\Gamma_{i}$ is the relaxation frequency which characterizes various relaxation modes that include relaxations due to the translational diffusion, rotational diffusion and bending. The relative mode strength (amplitude) of the $i$ th relaxation mode is $A_{i}$. For the present case, centre of mass diffusion is the dominant process and $\Gamma_{i}$ has been identified as $\Gamma_{i}=D_{i} q^{2}$, where the translational diffusion coefficient of the $i$ th particle is $D_{i}$. The expression for $g_{1}(t)$ remains valid for polydisperse samples and for a situation where the relaxation frequency distribution has several peaks. Further details of this discussion can be found elsewhere (Pecora 1985). According to Einstein relation, the $\bar{D}_{\mathrm{o}}$ (the $z$-average diffusion coefficient at infinite dilution) is inversely proportional to the translational frictional coefficient, $f_{\mathrm{t}}$, at infinite dilution by the relation

$$
\bar{D}_{\mathrm{o}}=\frac{k_{\mathrm{B}} T}{f_{\mathrm{t}}}=D=\frac{k_{\mathrm{B}} T}{6 \pi \eta R_{\mathrm{h}}},
$$

where $k_{\mathrm{B}}$ is the Boltzmann constant and $T$ the absolute temperature, $R$ the hydrodynamic radius and $\eta$ the solution viscosity. It is appropriate to refer to $R$ as effective radius.

The electrophoretic mobility measurements were performed on samples containing ethanol as a function of 
ethanol volume fraction. The instrument used was Zeecom2000 (Microtec Corporation, Japan) zeta-sizer that permitted direct measurement of electrophoretic mobility and its distribution. The electrophoretic cell was made of teflon (dimensions, $140 \mathrm{~W} \times 40 \mathrm{D} \times 60 \mathrm{H} \mathrm{mm}$ ) with distance between electrodes fixed at $10 \mathrm{~cm}$. In all our measurements the migration voltage was fixed at $30 \mathrm{~V}$. The instrument was calibrated against $10^{-4} \mathrm{M}$ AgI aqueous solution. In a bid to calibrate the instrument independently, we carried out iso-electric $\mathrm{pH}(\mathrm{pI})$ measurements on gelatin solutions and obtained $\mathrm{pI}=5 \cdot 2 \pm 0 \cdot 4$, consistent with the nominal value.

\section{Results and discussion}

\subsection{Analysis of DLS data}

The initial conditions spelt out in Brilliantov et al (1996) model are sufficiently met in our experiment, thus the formalism described above is a suitable model for the analysis of the following experimental results pertaining to assembly on 2-D substrates (Mohanty and Bohidar 2005). The corresponding particle size distribution (figure 1) was estimated from dynamic light scattering data. The wide particle size distribution is consistent with the requirement of (2) and least-squares fitting yields $\alpha=4 \cdot 01$. The corresponding AFM picture is shown in the inset of figure 1 .

However, in 3-D, Smoluchowski aggregation kinetics, which is a versatile and powerful tool to model aggregation phenomenon, has been successfully used to describe time-dependent evolution of aggregate size in bio-polymeric systems (Feder et al 1984). In an earlier work (Bohidar and Jena 1993), involving sol-gel transition in aqueous gelatin solutions, the Smoluchowski aggregation kinetics adequately described the temporal growth of gelatin undergoing gel transition. In this model, the temporal evolution of size distribution, $n_{i}(\tau)$, is given by (Smoluchowski 1916, 1917)

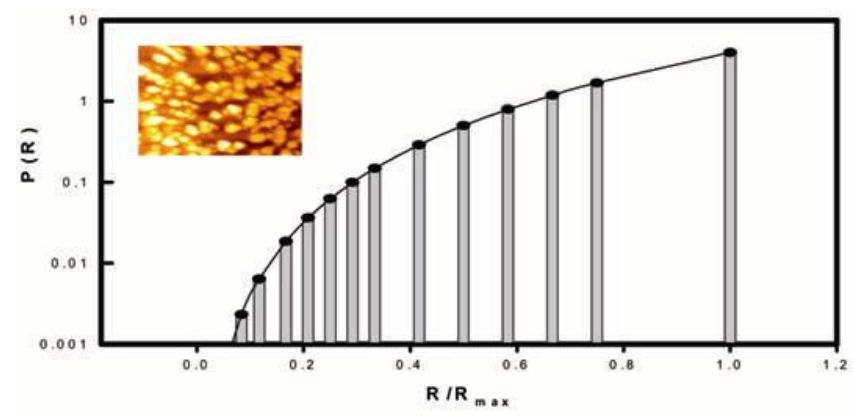

Figure 1. Dynamic light scattering data of particle size distribution of the gelatin sample. Fitting to (2) gives $\alpha=4.01$. Inset shows the corresponding AFM picture of the solution (Mohanty and Bohidar 2005)

$$
\frac{\mathrm{d} n_{k}}{\mathrm{~d} \tau}=\sum_{i+j=k=2}^{\infty} n_{i} A_{i j} n_{j}-2 \sum_{j=1}^{\infty} n_{k} A_{k j} n_{j} .
$$

Here, the reduced time, $\tau$, is defined through collision rate constant $\left(\gamma_{\mathrm{s}}\right)$ as $\tau=\gamma_{\mathrm{s}} \varepsilon t$. Smoluchowski assumed that the $i$ mer and $j$-mer bind irreversibly on collision and the probability of their collision is given by $\varepsilon$ (also called sticking coefficient). The transition matrix, $A_{i j}$, is given as (Feder et al 1984, 1985)

$$
A_{i j}=\frac{\left(D_{i}+D_{j}\right)\left(R_{i}+R_{j}\right)}{4 D_{0} R_{0}} .
$$

Smoluchowski assumed $A_{i j} \approx 1$ and came up with a cluster size distribution as a function of time, $\tau$, as (Feder et al 1984, 1985)

$$
n_{i}=\frac{\tau^{i-1}}{(1+\tau)^{i+1}}, i=1,2,3 .
$$

In this picture, the clusters grow with a power law behaviour where the radius of $i$ th cluster (a cluster with $i$-monomers in it) will show $R_{i} \sim i^{\beta}$ with $\beta=1 / d_{\mathrm{f}}$. The cluster exponent $\beta=1 / 3$ for spherical closely packed cluster, $1 / 2$ for a linear random coil polymer-like cluster and $6 / 5 D$ for diffusion limited clusters, where $D$ is Eucleadian dimension.

The intensity of the light scattered from such a system will be (Feder et al 1984, 1985)

$$
I_{\mathrm{s}}=I_{0} \sum_{i} n_{i} i^{2}
$$

And the same for radius of the aggregate will be given as (Feder et al 1984, 1985)

$$
R_{\mathrm{h}}^{2}=\sum_{i} n_{i} R_{i}^{2} i^{2}
$$

where $R_{0}$ and $I_{0}$ are radius of the monomer and intensity of light scattered by monomers in solution. Equations (13) and (14) with (12) will yield (Feder et al 1984, 1985)

$$
I_{\mathrm{s}}=I_{0}(1+2 \tau)
$$

and

$$
R_{\mathrm{h}}=R_{0}(1+\tau),
$$

where $R_{0}$ is $R_{\mathrm{h}}$ at $\tau=0$. A more generalized approach gives

$$
I_{\mathrm{s}}=I_{0}\left(1+\Gamma_{\mathrm{I}} \tau\right)^{\delta_{\mathrm{I}}}
$$

and

$$
R_{\mathrm{h}}=R_{0}\left(1+\Gamma_{\mathrm{R}} \tau\right)^{\delta_{\mathrm{R}}},
$$

where $\Gamma_{\mathrm{I}}$ and $\Gamma_{\mathrm{R}}$ are intensity and radius growth rate parameters. $\delta_{\mathrm{R}}$ can be approximated to the cluster exponent, 
$\beta=1 / d_{\mathrm{f}}$. However, for $\delta_{\mathrm{I}}$ no such clear relationship can be established. The temporal growth of aggregates in the bulk of the solution was measured through intensity of light scattered $\left(I_{\mathrm{s}}\right)$ at $90^{\circ}$ and effective hydrodynamic radius, $R_{\mathrm{h}}$. The variations are shown in figure 2 . The corresponding polydispersity that characterizes the width of the particle size distribution was found to be rather narrow (data not shown).

The experimental data could not be fitted to (15) and (16). In fact, the temporal growth of hydrodynamic radius, $R_{\mathrm{h}}(t)$ and scattered intensity, $I_{\mathrm{s}}(t)$, measured in the bulk were observed to exhibit $R_{\mathrm{h}} \sim t^{z}$ and $I_{\mathrm{s}} \sim t^{z}$ with $z=1 / d_{\mathrm{f}}$, giving a fractal dimension, $d_{\mathrm{f}}$, in $3-\mathrm{D} \approx 2 \cdot 6 \pm 0 \cdot 2$. Based on this, one can argue that in (15) and (16) the second term in the bracket is the dominant term $\left(\Gamma_{\mathrm{R}} \tau\right.$ and $\left.\Gamma_{\mathrm{I}} \tau>>1\right)$ giving the observed power-law features to measured $R_{\mathrm{h}}$ and $I_{\mathrm{s}}$ vs $t$ data. A second inference is that though Smoluchowski aggregation model adequately describes the gelation kinetics in this biopolymer its applicability to selfassociation remains rather qualitative (Bohidar and Jena 1993).

During the association process, the highly polydisperse aggregates are driven towards a monodisperse solution (The data fitting yielded polydispersity, $P \sim t^{-0 \cdot 2}$ ). DLA processes are known to produce smaller aggregates formed rapidly with wide particle size distribution. In contrast, RCA aggregates grow slowly into large clusters and exhibit low polydispersity in size distribution. The fractal dimension observed in the bulk $\left(d_{\mathrm{f}}\right.$ in $\left.3-\mathrm{D} \approx 2 \cdot 6 \pm 0 \cdot 2\right)$ is close to that of DLA whereas the polydispersity feature and aggregate size growth mimics that of a RLA process, which appears anomalous.

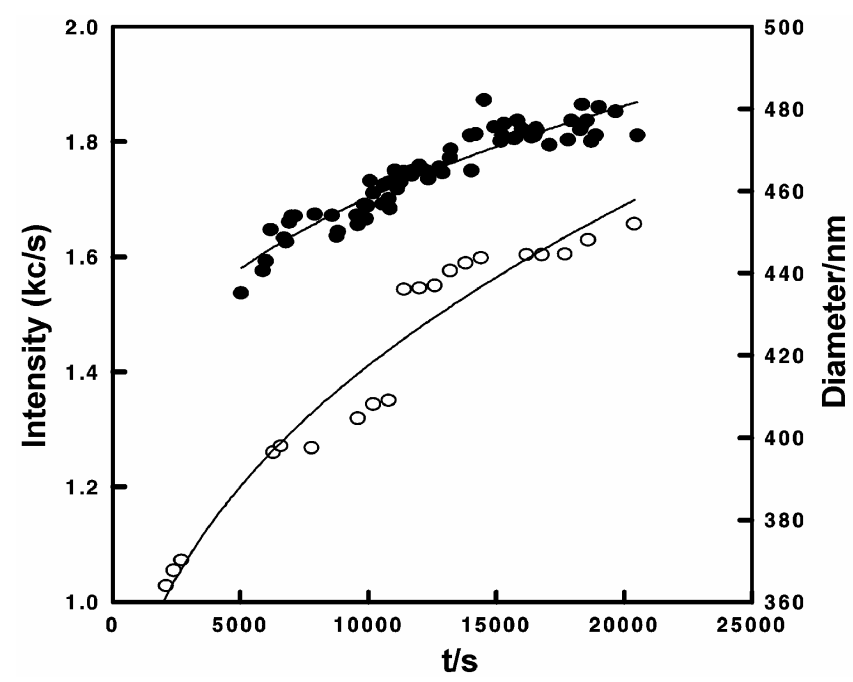

Figure 2. Temporal growth of effective diameter, $R_{\mathrm{h}}(t)(\mathrm{O})$ and intensity $(\bullet)$ of light, $I_{\mathrm{s}}(t)$, scattered from aggregated gelatin molecules in aqueous solutions measured by dynamic light scattering. The data fitting to $R_{\mathrm{h}}(t) \sim I_{\mathrm{s}}(t) \sim t^{1 / d_{\mathrm{f}}}$ gave $d_{\mathrm{f}}=$ $2 \cdot 6 \pm 0 \cdot 2$

\subsection{Analysis of electrophoresis data}

The gelatin stock solutions were titrated with ethanol and the turbidity of the solution and electrophoretic mobility of aggregates was measured continuously. These profiles are shown in figure 3. A clear phase transition was observed at ethanol volume fraction $=0.47 \pm 0.03$. During this process inter-molecular soluble aggregates of gelatin molecules are formed. The electrophoretic mobility and zeta potential profiles of these aggregates are shown in figure 3 . It is clearly seen that close to the ethanol concentration $=0.47$ (notice sharp increase in turbidity in these figures signaling phase transition) and zeta potential showed a sharp drop in its values, which corresponds to the minimum mobility of the soluble aggregates. Some disagreement was seen at higher polypeptide concentration. We did observe charge neutralization (zeta potential and mobility minima) at ethanol volume fraction $\approx 0.47 \pm$ 0.02 , while turbidity maximum was seen at $\approx 0.37 \pm 0.02$ (data not shown). Thus, it can be clearly argued that higher gelatin concentration has profound effect on the measured zeta potential value that makes the $\mathrm{pI}$ shift by as much as $1.5 \mathrm{pH}$ units due to complex intermolecular interactions. The charge neutralization process is completely governed by screened Coulumbic interactions whereas the turbidity maximum arises from a complex interplay of various entropic and enthalpic processes. In our interaction model, we have not been able to address the issue of one to one correspondence between the turbidity and electrophoretic data, though we qualitatively argue that turbidity maximum must coincide with minimum zeta potential. This in turn implies minimum net charge associated with the soluble aggregates. Correspondingly, the zeta potential which was $\approx 6 \mathrm{mV}$ for gelatin molecules $(0 \cdot 01 \%(\mathrm{w} / \mathrm{v}))$ dropped to $\approx 0.23 \mathrm{mV}$ for soluble aggregates, a decrease of $\approx 97 \%$.

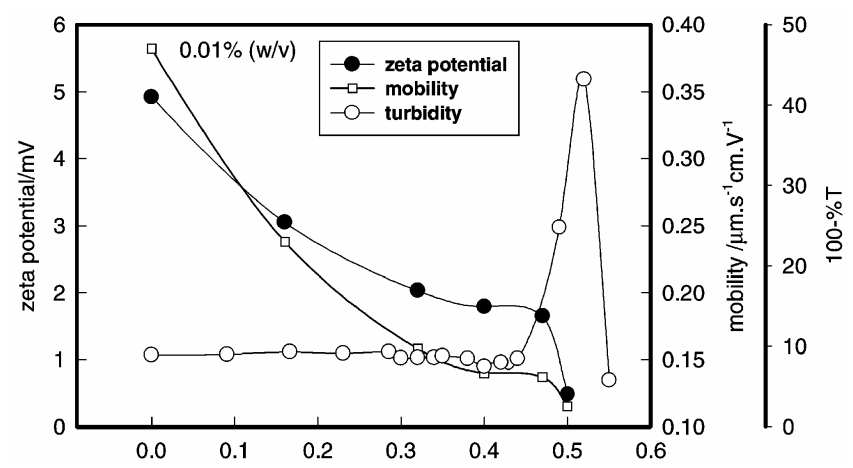

Figure 3. Variation of electrophoretic mobility, zeta potential and turbidity of aggregated gelatin molecules in aqueous solutions measured as a function of ethanol volume fraction. Notice that close to ethanol volume fraction $=0.47$ the turbidity becomes maximum and mobility (and zeta potential) becomes minimum implying significant charge neutralization in aggregates. 


\section{Conclusions}

The physical mechanism of aggregation in the bulk can be visualized as follows. Gelatin is not soluble in alcohols whereas water is a good solvent. As ethanol is added to water, the water molecules will preferentially bind to the alcohol molecules through hydrogen bonding and the resultant binary mixture becomes a marginal solvent for gelatin molecules. Secondly, the dielectric constant $(\varepsilon)$ decreases (Waller and Strang 1996) significantly facilitating stronger electrostatic interactions between charged segments (both intra and inter) of gelatin molecules. The strength of electrostatic interactions between two oppositely charged particles increases as $\varepsilon^{-3 / 2}$ at a given temperature as per the Debye-Huckel theory (Tanford 1967). Since the solution $\mathrm{pH}$ was close to the iso-electric point of gelatin, there is no net charge on the polypeptide. Nonetheless, as mentioned already, the chemical structure of this biopolymer indicates almost $1: 1$ positive and negatively charged patches on this linear random coil molecule. These overlap as the chain contracts due to the decrease in the FloryHuggin's solute-solvent interaction (Gupta and Bohidar 2005) brought in by the presence of ethanol resulting in bringing charged segments to each other's vicinity through electrostatic interactions yielding chain collapse. This is clearly observed from the electrophoresis data presented.

\section{Acknowledgement}

Authors acknowledge support from the Department of Science and Technology, Government of India, under its nano-initiative research program.

\section{References}

Birdi K S 1991 Fractals in chemistry, biochemistry and biophysics (New York: Plenum Press)

Bohidar HB 2002 in Handbook of polyelectrolytes-II (CA, USA: American Scientific Publishers) p. 117

Bohidar H B and Jena S S 1993 J. Chem. Phys. 988970

Brilliantov N V, Andrienko Y A, Krapivsky P L and Kurths J 1996 Phys. Rev. Lett. 764058

Bungenberg de Jong H G 1949 Colloid science (ed.) H R Kruyt (New York: Elsevier) Vol. II, p. 335

Di Biasio A, Bolle G, Cametti C, Codestefano P, Scortino F and Tartagalia P 1994 Phys. Rev. E50 1649

Evans J W 1993 Rev. Mod. Phys. 651281

Feder J, Jossang T and Rosenquist E 1984 Phys. Rev. Lett. 53 1403

Feder J, Jossang T and Rosenquist E 1985 J. Chem. Phys. 82 574

Gupta A and Bohidar H B 2005 Phys. Rev. E72 011507

Mohanty B and Bohidar HB 2003 Biomacromolecules 4 1080

Mohanty B and Bohidar H B 2005 Int. J. Polym. Mater. 54 675

Oparin A I 1957 The origin of life on earth (London: Oliver and Boyd)

Pecora R 1985 Dynamic light scattering (New York: Plenum)

Smoluchowski M V 1916 Phys. Z. 17557

Smoluchowski M V 1917 Phys. Z. 92129

Tanford C 1967 Physical chemistry of macromolecules (London: John-Wiley)

Witten Jr T A and Sander LM 1981 Phys. Rev. Lett. 47 1400

Witten Jr T A and Sander L M 1983 Phys. Rev. B27 5686

Waller R and Strang T J K 1996 Collection Forum 1270 